\title{
Symmetric Tight Frame Wavelets With Dilation Factor $M=4$
}

\author{
Farras Abdelnour \\ University of Pittsburgh, \\ Radiology Department, \\ 200 Lothrop Street, \\ Pittsburgh, PA 15213
}

\begin{abstract}
In this paper we discuss a new set of symmetric tight frame wavelets with the associated filterbank outputs downsampled by four at each stage. The frames consist of seven generators obtained from the lowpass filter using spectral factorization, with the lowpass filter obtained via a simple method using Taylor polynomials. The filters are simple to construct, and offer smooth scaling functions and wavelets. Additionally, the filterbanks presented in this paper have limited redundancy while maintaining the smoothness of underlying limit functions. The filters are linear phase (symmetric), FIR, and the resulting wavelets possess vanishing moments.
\end{abstract}

Key words: wavelet transform, frame, symmetric filterbanks, multiresolution analysis

Email address: farras.abdelnour@gmail.com (Farras Abdelnour) 


\section{Introduction}

Tight frame filterbanks are a generalization of orthogonal filterbanks. While the analysis and synthesis filterbanks are identical, they offer additional degrees of freedom, which can be exploited to improve or obtain certain properties of the limit functions such as smoothness and symmetry. This is particularly true for the case of frames with dilation factor $M=2$, where in the orthogonal case symmetry is not allowed in general (the exception being Haar wavelet), while tight frames allow symmetry and smooth limit functions.

Tight frames (TF) have been well documented, see for example $[1,2,3$, $4,5,6,7]$, and more recently [8]. An excellent overview of wavelet frames is offered in $[9,10]$. A majority of the current literature has considered the design of perfect reconstruction (PR) tight frame filterbanks with $M=2$. Tight frame filterbanks allow properties which otherwise can only be obtained in the biorthogonal case such as symmetry and smoothness. Earlier papers discussing tight frame design can be found in $[11,12]$ where 3 -channel tight frame minimum length filterbanks are obtained. Three channel symmetric TF filterbanks are discussed in $[13,14,15]$. Four channel symmetric TF filterbanks have been discussed in $[13,16,17,18,19,20,21]$. One of the drawbacks of tight frame filterbanks of $N$ filters $(N>2)$ based on $M=2$ is the resulting redundancy rate, which is $N-1$ for the one dimensional case. This can lead to a high redundancy as the number of filters in a filterbank increases. The case $M>2$ results in symmetric as well as orthogonal wavelets, see for example [22] where orthogonal symmetric filterbank design with $M=4$ is discussed. 
In [23] orthogonal and symmetric filterbank design for the case $M=8$ is presented, with application to image compression. A thorough discussion of orthogonal filterbanks for general $M$ can be consulted in [24, 25]. The disadvantages of orthogonal symmetric filterbanks are their underlying limit functions' lack of smoothness, and in general the filterbanks are shiftsensitive. This becomes an issue for example in image denoising where a shift of the input can alter the denoising performance. Wavelets based on symmetric biorthogonal filterbanks with $M>2$ have been considered, see for example [26] where the authors describe linear phase critically sampled $M$ band filterbank design using lattice structure. While the resulting synthesis filters lead to smooth limit functions, the corresponding analysis filters lack smoothness. As an alternative, filterbanks based on frames lead to filters with improved frequency selectivity for both the synthesis and analysis filterbanks as well as shorter filters (at the expense of redundant throughput). In [27] the authors outline properties of $M$-band tight frame filterbanks, but do not offer a design procedure.

In [28], Tanaka discusses construction of FIR-based dual frames for any scaling factor $M$. Given an analysis filterbank, the paper describes the design of a synthesis filterbank frame given the analysis filterbank. The resulting synthesis FB can be optimized according to certain criteria. The approach requires an a priori knowledge of the analysis filterbank. In [29], compactly supported multivariate wavelet dual frames with minimal support have been constructed for any integer dilation matrix.

In [17] and [30] symmetric frames are constructed with the underlying wavelets possessing a high number of vanishing moments while remaining 
FIR, smooth, and maintaining short support. The resulting filterbanks require pre/post processing with IIR filter. In a more recent paper [31], 6-band tight frame filterbanks with scaling factor $M=4$, and with symmetric lowpass filter, are designed using Gröbner basis method [32, 33]. In [34], the authors propose $n$-dimensional tight frames obtained from orthogonal filterbanks by properly reducing the downsampling rate. This elegant approach does not benefit from the fact that filterbanks designed as frames have shorter supports. In [35] a symmetric dual frame wavelet system is designed with $M=4$ and six bands. The resulting limit functions are smooth and benefit from short supports. Multivariate dual frames based on the mixed oblique extension principle with six wavelets and $M=4$ are discussed in [36]. In [37] the authors present complex symmetric orthonormal wavelets based on FIR filters with $M=4$. A general method for constructing $\mathrm{TF}$ and orthogonal wavelets with any integer scaling factor $M$ is described by Petukhov in [38]. It addresses the design of both even and odd lengths linear phase filterbanks. While [38] discusses the structure of the matrix containing the filters, it does not show how to obtain the lowpass filter from which the remaining filters are generated. The paper outlines the steps leading to generating an orthogonal filterbank as well as tight frames with $M$ wavelets but does not give explicit examples of the filterbanks.

In this article, we build upon [38] and describe the construction of a tight frame (anti-)symmetric filterbank with $M=4$ and eight filters ( 7 wavelets). We start by describing the design of the lowpass filter first, then using spectral factorization methods we generate the remaining filters. 


\subsection{Paper's Contribution}

We use the truncated Taylor series technique for the lowpass filter, in addition to spectral factorization for the remaining filters as design methods. Lowpass filter design based on Taylor series has been considered in [39] and later in [25]. The proposed method is also closely related to the complex orthogonal symmetric wavelet design method given recently by Han [40]. We

seek to design tight frame (anti-)symmetric filterbanks $\left\{h_{0}, h_{1}, h_{2}, h_{3}, h_{4}, h_{5}, h_{6}, h_{7}\right\}$

of even length with $M=4$. The design approach leads first to a lowpass filter $h_{0}$ found via Taylor polynomials method. Three more filters $\left\{h_{3}, h_{4}, h_{7}\right\}$ are then found by modulating $h_{0}$, leading to one highpass filter and two bandpass filters. The remaining filters $\left\{h_{1}, h_{2}, h_{5}, h_{6}\right\}$, all bandpass, are found via a spectral factorization approach involving two equations. The solutions of the equations result in two polynomials which are then used as polyphase components to construct the filters. All filters are assumed to be of even lengths, with filter lengths $4 L$ and $4 L+2, L \in \mathbb{N}$, treated separately.

\subsection{Advantages of Proposed Wavelets}

One possible drawback of wavelet frames with $M=2$ is the extent of redundancy of the resulting wavelet coefficients, which can be computationally demanding. By using a higher scaling factor ( $M=4$ in this case) for wavelet frames, we reduce the redundancy by a factor of three (from $N-1$ to $(N-1) / 3)$ for the case of $1-D$ signals, while at the same time offering an alternate frequency resolution of the resulting filterbank. Thus we benefit from the advantages of redundancy while keeping the necessary computation (performing the transform and its inverse) manageable. 


\subsection{Organization}

In Section 2 we briefly discuss the basics of tight frame filterbanks under $M=4$. We discuss the essentials of tight frame filterbanks for the case $M=4$, both from filters as well as polyphase perspectives. In Section 3 we describe the design and construction of a filterbank consisting of eight (anti)symmetric filters starting with designing the lowpass filter. In Section 4 we present examples of filterbanks of various lengths, approximation orders, and vanishing moments. Section 5 presents an application example in signal denoising using proposed wavelets. Section 6 comments on the smoothness of the wavelets. We conclude the paper with Section 7 .

\subsection{Notations}

A filterbank of 8 bands is notated as $\left\{h_{0}, h_{1}, h_{2}, h_{3}, h_{4}, h_{5}, h_{6}, h_{7}\right\}$, where the index in $h_{i}$ refers to a progressively higher passband, such that $h_{0}$ refers to a lowpass filter, and $h_{7}$ represents a highpass filter. Bold capital letters are reserved for matrices. Scaling functions are represented by $\phi(\cdot)$, while wavelets are given by $\psi_{i}(\cdot)$. In frequency domain, filters are given by $H_{i}(z) . H_{i}(\omega)$ is reserved for the $i$ th filter evaluated on the unit circle $e^{\jmath \omega} \cdot H_{i j}(\cdot)$ represents the $j$ th polyphase component of the $i$ th filter. All the filters discussed in this paper take on the form $H_{0}(z)=\left(1+z^{-1}\right)^{k}\left(1+z^{-1}+z^{-2}+z^{-3}\right)^{K_{0}} Q_{0}(z)$, $k \in\{0,1\}$, for the lowpass filter and $H_{i}(z)=\left(1-z^{-1}\right)^{K_{i}}\left(1+z^{-1}\right)^{L_{i}} Q_{i}(z)$ for the bandpass and highpass filters (in the latter case we have $L_{i}=0$ ), and $1 \leq i \leq 7$. 


\section{Properties and Conditions}

In this section, we discuss definitions and properties pertaining to the case of $M=4$ tight frame filterbanks. A set of $N-1$ univariate real wavelets $\left\{\psi_{i}\left(4^{m} \cdot-n\right), i=1 \ldots N-1\right\}$, where $\{m, n\} \subset \mathbb{Z}$, constitutes a frame when for $0<A \leq B<\infty$ and any function $f \in L_{2}(\mathbb{R})$ we have

$$
A\|f\|^{2} \leq \sum_{i=1}^{N-1} \sum_{m, n}\left|\left\langle f, \psi_{i}\left(4^{m} \cdot-n\right)\right\rangle\right|^{2} \leq B\|f\|^{2},
$$

where $A$ and $B$ are known as frame bounds, and the inner product $\langle\cdot, \cdot\rangle$ defined as follows:

$$
\langle f, g\rangle=\int_{-\infty}^{\infty} f(t) \bar{g}(t) \mathrm{d} t .
$$

The special case of $A=B$ is known as tight frame. In general, a wavelet system consisting of one scaling function $\phi(t)$ and $N-1$ wavelets with dilation $4,4 \leq N$, defines the following spaces:

$$
\begin{aligned}
\mathcal{V}_{j} & =\underset{n}{\operatorname{Span}}\left\{\phi\left(4^{j} t-n\right)\right\}, \\
\mathcal{W}_{i, j} & =\operatorname{Span}_{n}\left\{\psi_{i}\left(4^{j} t-n\right)\right\}, i=1 \ldots N-1
\end{aligned}
$$

with

$$
\mathcal{V}_{j}=\mathcal{V}_{j-1} \cup \mathcal{W}_{1, j-1} \cup \mathcal{W}_{2, j-1} \cup \ldots \cup \mathcal{W}_{N-1, j-1}
$$

and $t \in \mathbb{R}$. The corresponding scaling function and wavelets satisfy the following multiresolution equations:

$$
\begin{aligned}
\phi(t) & =2 \sum_{n} h_{0}(n) \phi(4 t-n), \\
\psi_{i}(t) & =2 \sum_{n} h_{i}(n) \phi(4 t-n), i=1 \ldots N-1 .
\end{aligned}
$$


It is shown in [41] and [42] that for almost any tight wavelet frame, one has $\mathcal{W}_{0}=\mathcal{V}_{1}$, where the closure of the spaces is taken in $L_{2}(\mathbb{R})$. The frame bounds $A$ and $B$ take on the value [3]

$$
A=B=\frac{1}{4} \sum_{n=0}^{N-1}\left\|h_{n}\right\|^{2} .
$$

A function $f \in L_{2}(\mathbb{R})$ can then be expanded in terms of the wavelets and scaling function as follows:

$$
f(t)=\sum_{k}\left\langle f, \phi_{0, k}(t)\right\rangle \phi_{0, k}(t)+\sum_{i=1}^{N-1} \sum_{j=0}^{\infty} \sum_{k}\left\langle f, \psi_{i, j, k}(t)\right\rangle \psi_{i, j, k}(t),
$$

where $\phi_{0, k}(t)=\phi(t-k), \psi_{i, j, k}(t)=\psi_{i}\left(4^{j} t-k\right)$. The filters discussed in this article are all of finite length (FIR), and the resulting scaling function $\phi(\cdot)$ and wavelets are of finite support.

\subsection{Oversampled Filterbanks}

For reasons to be made clear in Section 3, we need to address the oversampled polyphase filters [43]. Given a set of $N$ filters with a dilation factor 4 , define the filters in terms of their polyphase components $H_{i j}(z)$ :

$$
H_{i}(z)=\sum_{j=0}^{3} z^{-j} H_{i j}\left(z^{4}\right)
$$

where

$$
H_{i j}(z)=\sum_{n} h_{i}(4 n-j) z^{-n} .
$$

Now define the polyphase analysis matrix as

$$
\mathbf{H}(z)=\left(\begin{array}{ccc}
H_{0,0}(z) & \ldots & H_{0,3}(z) \\
H_{1,0}(z) & \ldots & H_{1,3}(z) \\
\vdots & \ddots & \vdots \\
H_{N-1,0}(z) & \ldots & H_{N-1,3}(z)
\end{array}\right) .
$$


If we define a signal $X(z)$ in terms of its polyphase components we have

$$
\chi(z)=\left[\begin{array}{llll}
X_{0}(z) & X_{1}(z) & X_{2}(z) & X_{3}(z)
\end{array}\right]^{T},
$$

with $X_{j}(z), 0 \leq j \leq 3$, defined in terms of the time domain signal $x(n)$ as follows

$$
X_{j}(z)=\sum_{n} x(4 n-j) z^{-n} .
$$

Then the overall output signal $\widehat{X}(z)$ of the analysis/synthesis filterbanks can be expressed as

$$
\widehat{X}(z)=\left[\begin{array}{llll}
1 & z^{-1} & z^{-2} & z^{-3}
\end{array}\right] \mathbf{H}^{T}\left(z^{4}\right) \mathbf{H}\left(z^{-4}\right) \chi\left(z^{4}\right),
$$

and to meet PR condition $\widehat{X}(z)=X(z)$ we require that [44]

$$
\mathbf{H}^{T}(z) \mathbf{H}\left(z^{-1}\right)=\mathbf{I},
$$

and we obtain

$$
\sum_{k} H_{k, i}(z) H_{k, j}\left(z^{-1}\right)= \begin{cases}1 & \text { if } i=j=0 \\ 0 & \text { otherwise }\end{cases}
$$

The frame conditions discussed above can be expressed in terms of oversampled filterbanks. Given a set of $N$ filters with a dilation factor 4 (with $N \geq 4$ ), the PR condition can be written as follows:

$$
\begin{gathered}
\sum_{n=0}^{N-1} H_{n}(z) H_{n}(1 / z)=4 \\
\sum_{n=0}^{N-1} H_{n}(-z) H_{n}(1 / z)=0 \\
\sum_{n=0}^{N-1} H_{n}(\jmath z) H_{n}(1 / z)=0 \\
\sum_{n=0}^{N-1} H_{n}(-\jmath z) H_{n}(1 / z)=0,
\end{gathered}
$$


where $\jmath=\sqrt{-1}$. Above equations can be put in matrix form using the modulation matrix given by [44]:

$$
\mathbf{H}_{m}(z)=\left(\begin{array}{cccc}
H_{0}(z) & H_{1}(z) & \ldots & H_{N-1}(z) \\
H_{0}(\jmath z) & H_{1}(\jmath z) & \ldots & H_{N-1}(\jmath z) \\
H_{0}(-z) & H_{1}(-z) & \ldots & H_{N-1}(-z) \\
H_{0}(-\jmath z) & H_{1}(-\jmath z) & \ldots & H_{N-1}(-\jmath z)
\end{array}\right),
$$

resulting in

$$
\mathbf{H}_{m}(z) \mathbf{H}_{m}^{T}(1 / z)=4 \mathbf{I}
$$

It has been shown in [30] and [45] that the filters embodying a tight frame filterbank $\left\{h_{i}\right\}_{i=0}^{N-1}$ must satisfy the following condition:

$$
\sum_{n=0}^{3}\left|H_{i}\left(\omega+\frac{2 \pi n}{4}\right)\right|^{2} \leq 4, i=0 \ldots N-1 .
$$

\subsection{Constraints on the Length of $h_{0}$}

It is shown in [11] that a 3-band tight frame with dilation factor 2 and satisfying PR the minimum length of the lowpass filter $h_{0}$ is subject to the condition

$$
\text { length } h_{0} \geq K_{0}+\min \left(K_{1}, K_{2}\right)
$$

where $K_{0}$ is the number of zeros at $z=-1$ for the filter $H_{0}(z)$ and $K_{i}$ is the number of zeros at $z=1$ for the filters $H_{i}(z), i=1,2$. In [18] the minimum length for the symmetric case is given by

$$
\text { length } h_{0} \geq K_{0}+2 \min \left(K_{1}, K_{2}, \ldots K_{N-1}\right)-1
$$

It is straight forward to extend condition (10) to the case of $M=4$, in which case we obtain for $h_{0}$ 's minimum length

$$
\text { length } h_{0} \geq 3 K_{0}+2 \min \left(K_{1}, K_{2}, \ldots, K_{N-1}\right)-1 \text {. }
$$




\subsection{Redundancy}

A tight-frame analysis system generates more data at the output than the input. The redundancy rate depends on both $M$ and $N$, as well as the number of filtering stages $l$. As previously shown in [46], for a single stage filterbank the redundancy ratio is $R=\frac{N}{M}$. For multiple stages we have the contribution due to the lowpass filter and its following stages in addition to the highpass/bandpass filters. As such, the redundancy ratio at the $i^{\text {th }}$ stage is $\frac{N-1}{M^{i}}$ in addition to the lowpass filter contribution, $\frac{1}{M^{i}}$. At the analysis output the latter is just $\frac{1}{M^{l}}$. Putting the above results together we have

$$
\begin{aligned}
R & =(N-1)\left(\frac{1}{M}+\frac{1}{M^{2}}+\ldots+\frac{1}{M^{l-1}}\right)+\frac{N}{M^{l}} \\
& =\frac{N-1}{M-1}\left(1-\frac{1}{M^{l-1}}\right)+\frac{N}{M^{l}} .
\end{aligned}
$$

From the last equation we have $R \rightarrow \frac{N-1}{M-1}$ as $l \rightarrow \infty$ and $R=\frac{N}{M}$ for $l=1$. For the case $M=4$ the redundancy $R$ is bounded by $\frac{N}{4} \leq R \leq \frac{N-1}{3}$, and with $N=8$ we have $2 \leq R \leq \frac{7}{3}$.

\subsection{Smoothness of the scaling function}

One of the advantages of tight frame filterbanks is the possibility of achieving a high degree of smoothness $\nu_{2}$. The orthogonal case, on the other hand, has typically low smoothness for a given $K_{0}$. It is shown in [47] that shift stability, while true for orthogonal wavelets, extends to tight frames as well. The authors show that the property of tight frame decomposition with scaling factor $M$ of functions in $L_{2}(\mathbb{R})$ can be extended in a stable way to functions in Sobolev spaces when the limit functions of the tight frames meet mild regularity and vanishing moment conditions. Smoothness is measured 
using the Sobolev exponent of a scaling function $\phi$ defined as $[48,49]$ :

$$
\nu_{2}(\phi):=\sup \left\{\nu_{2}: \int_{-\infty}^{\infty}|\Phi(\omega)|^{2}\left(1+|\omega|^{2}\right)^{\nu_{2}} \mathrm{~d} \omega<\infty\right\} .
$$

The actual computation of $\nu_{2}$ is found using [22], and for the normalization $\sum_{n} h_{0}(n)=2$ we have

$$
\nu_{2}=-\frac{1}{2} \log _{4} \lambda_{\max }
$$

where $\lambda_{\max }$ is the largest eigenvalue of a matrix generated by $\left(c_{2 i-j}\right)_{-N \leq i, j \leq N}$ with $c(z)=Q_{0}(z) Q_{0}\left(z^{-1}\right)$ and $Q_{0}(z)$ known from

$$
H_{0}(z)=\left(1+z^{-1}\right)^{k}\left(1+z^{-1}+z^{-2}+z^{-3}\right)^{K_{0}} Q_{0}(z), k \in\{0,1\} .
$$

The lowpass filter's structure and properties are described in the design section that follows.

\section{Filterbank Design}

In this section, we discuss the design of a filterbank consisting of eight (anti-)symmetric filters, starting with a separately designed lowpass filter $h_{0}$ of even length.

\subsection{Lowpass Filter Design}

The lowpass filter in a tight frame filterbank with $M=4$ must satisfy two conditions:

$$
\begin{aligned}
\left(1+z^{-1}+z^{-2}+z^{-3}\right)^{K_{0}} & \mid H_{0}(z) \\
\left(2-z^{2}-z^{-2}\right)^{K_{\min }} & \mid 4-H_{0}(z) H_{0}\left(z^{-1}\right)
\end{aligned}
$$

where $K_{\min }=\min \left(K_{1}, K_{2}, \ldots, K_{N-1}\right)$. The first condition guarantees the reproduction of polynomials of degree up to and including $K_{0}-1$, while 
the second condition guarantees that each highpass or bandpass filter has a minimum of $K_{\min }$ zeros at $z=1$, a necessary condition for wavelet vanishing moments. The filter is of even length and takes on the following general form:

$$
H_{0}(z)=\left(1+z^{-1}\right)^{k}\left(1+z^{-1}+z^{-2}+z^{-3}\right)^{K_{0}} Q_{0}(z),
$$

where $Q_{0}(z)$ is a symmetric polynomial of length $2 K_{\min }-1$, and the factor $\left(1+z^{-1}\right)^{k}$ serves to preserve the filter's even length, such that $k=1$ for the case where $K_{0}$ is even, and $k=0$ otherwise. We base the proposed design method on the earlier work by Herrmann [39]. The proposed method is also closely related to the complex orthogonal symmetric wavelet design method given recently by Han [40]. We first consider the case $k=1$ corresponding to $K_{0} \in 2 \mathbb{N}$. Following [39], we perform a change of variables preserving the filter's symmetry:

$$
x=\frac{-z^{-1}+2-z}{4} .
$$

The filter $H_{0}(z)$ is then converted to a function $P(x)$ reflecting the conditions $(12,13)$ at $z=1, z=-1$, and $z= \pm \sqrt{-1}$ respectively corresponding to $x=0, x=1$, and $x=\frac{1}{2}$. With $K_{0}=2 K$ we obtain the following identities:

$$
\begin{aligned}
P(0) & =1, \\
P^{(i)}(0) & =0, i=1 \ldots K_{\min }-1, \\
P^{(i)}(1) & =0, i=0 \ldots K-1, \\
P^{(i)}\left(\frac{1}{2}\right) & =0, i=0 \ldots 2 K .
\end{aligned}
$$

The filter's even length necessitates a factor of the form $\sqrt{1-x}$, so $P(x)$ takes on the form

$$
P(x)=A(x) \sqrt{1-x}(1-x)^{K}\left(\frac{1}{2}-x\right)^{2 K},
$$


where the polynomial $A(x)$ is of length $K_{\min }$ corresponding to the polynomial $Q_{0}(z)$. Taking into account flatness at $z=1$ corresponding to equation (15), $P(x)$ must satisfy the condition

$$
1-P(x)=B(x) x^{K_{\min }}
$$

Combining equations (16) and (17) we obtain the condition

$$
A(x) \sqrt{1-x}(1-x)^{K}\left(\frac{1}{2}-x\right)^{2 K}+B(x) x^{K_{\min }}=1 .
$$

The polynomial $A(x)$ is then given by

$$
\begin{aligned}
A(x) & =\frac{1-B(x) x^{K_{\min }}}{(1-x)^{K+\frac{1}{2}}\left(\frac{1}{2}-x\right)^{2 K}} . \\
& =\sum_{n=0}^{K_{\min }-1} c_{n} x^{n}+\mathcal{O}\left(|x|^{K_{\min }}\right) .
\end{aligned}
$$

Since $A(x)$ is of degree $K_{\min }-1$, the above expression reduces to

$$
A(x)=\sum_{n=0}^{K_{\min }-1} c_{n} x^{n} .
$$

Thus, $A(x)$ is obtained from a truncated Taylor series such that

$$
\frac{1}{(1-x)^{K+\frac{1}{2}}\left(\frac{1}{2}-x\right)^{2 K}}=A(x)+\mathcal{O}\left(|x|^{K_{\text {min }}}\right) .
$$

In other words, $A(x)$ is the Taylor polynomial resulting from expanding expression (19) around $x=0$ and keeping only the first $K_{\min }$ terms. The corresponding polynomial $Q_{0}(z)$ is then obtained by mapping back from $x$ to $z$. Notice that in the derivation of $A(x)$ there was no need to explicitly find $B(x)$. For the case $k=0\left(K_{0} \in 2 \mathbb{N}+1\right)$ a similar derivation results in $A(x)$ obtained from the Taylor polynomial of degree $K_{\min }-1$ :

$$
\frac{1}{(1-x)^{K+\frac{1}{2}}\left(\frac{1}{2}-x\right)^{2 K+1}}=A(x)+\mathcal{O}\left(|x|^{K_{\text {min }}}\right),
$$


where $K_{0}=2 K+1$. The polynomials $A(x)$ and $Q_{0}(z)$ are related as follows:

$$
Q_{0}(z)=A\left(\frac{-z^{-1}+2-z}{4}\right),
$$

from which we obtain the lowpass filter in equation (14). The proposed method is simple to implement and is a function of $K_{0}$ and $K_{\min }$. We next obtain the remaining filters $\left\{h_{i}\right\}_{i=1}^{7}$.

\subsection{Finding Filters $\left\{h_{3}, h_{4}, h_{7}\right\}$}

Having found $h_{0}$, we obtain filters $\left\{h_{3}, h_{4}, h_{7}\right\}$ by modulating $h_{0}$, similar to [18]. We first express $H_{0}(z)$ in terms of its polyphase components, as defined in equation (2). For the case of length $h_{0}=4 L$, and taking into account the symmetry of the filter, we have:

$$
H_{0}(z)=H_{00}\left(z^{4}\right)+z^{-1} H_{01}\left(z^{4}\right)+z^{-2-4 L} H_{01}\left(1 / z^{4}\right)+z^{-3-4 L} H_{00}\left(1 / z^{4}\right) .
$$

It should be verified that the resulting filter satisfies inequality (8). Notice how the structure of $H_{0}(z)$ in equation (21) leads to a symmetric filter. The filters $\left\{h_{3}, h_{4}, h_{7}\right\}$ are found as follows:

$$
\begin{aligned}
H_{3}(z)= & H_{00}\left(z^{4}\right)-z^{-1} H_{01}\left(z^{4}\right)+ \\
& z^{-2-4 L} H_{01}\left(1 / z^{4}\right)-z^{-3-4 L} H_{00}\left(1 / z^{4}\right), \\
H_{4}(z)= & H_{00}\left(z^{4}\right)+z^{-1} H_{01}\left(z^{4}\right)- \\
& z^{-2-4 L} H_{01}\left(1 / z^{4}\right)-z^{-3-4 L} H_{00}\left(1 / z^{4}\right), \\
H_{7}(z)= & H_{00}\left(z^{4}\right)-z^{-1} H_{01}\left(z^{4}\right)- \\
& z^{-2-4 L} H_{01}\left(1 / z^{4}\right)+z^{-3-4 L} H_{00}\left(1 / z^{4}\right) .
\end{aligned}
$$

Similarly, for the case length $h_{0}=4 L+2, H_{0}(z)$ is written as follows:

$$
H_{0}(z)=H_{00}\left(z^{4}\right)+z^{-1-4 L} H_{00}\left(1 / z^{4}\right)+z^{-2} H_{02}\left(z^{4}\right)+z^{-3-4 L} H_{02}\left(1 / z^{4}\right) .
$$


The remaining filters $\left\{h_{3}, h_{4}, h_{7}\right\}$ are then found by modulation, similar to equations (22-24). We next consider finding the remaining filters $\left\{h_{1}, h_{2}, h_{5}, h_{6}\right\}$ using spectral factorization. We consider separately the cases of length $h_{0}=$ $4 L$ and length $h_{0}=4 L+2, L \in \mathbb{N}$.

\subsection{Finding Bandpass Filters $\left\{h_{1}, h_{2}, h_{5}, h_{6}\right\}$ With Length $4 L$}

We look for the bandpass filters $\left\{h_{1}, h_{2}, h_{5}, h_{6}\right\}$ assuming the lowpass filter is of length $4 L, L \in \mathbb{N}$. Suppose the filter $h_{2}$ can be written as follows:

$$
H_{2}(z)=a\left(z^{4}\right)+z^{-1} b\left(z^{4}\right)+z^{-2-4 L} b\left(1 / z^{4}\right)+z^{-3-4 L} a\left(1 / z^{4}\right),
$$

where $a$ and $b$ are some (to be determined) polynomials in $z$. This guarantees $h_{2}$ to be a symmetric filter. Suppose moreover that the remaining filters are written as follows:

$$
\begin{aligned}
& H_{1}(z)=-a\left(z^{4}\right)+z^{-1} b\left(z^{4}\right)-z^{-2-4 L} b\left(1 / z^{4}\right)+z^{-3-4 L} a\left(1 / z^{4}\right), \\
& H_{5}(z)=-a\left(z^{4}\right)+z^{-1} b\left(z^{4}\right)+z^{-2-4 L} b\left(1 / z^{4}\right)-z^{-3-4 L} a\left(1 / z^{4}\right), \\
& H_{6}(z)=-a\left(z^{4}\right)-z^{-1} b\left(z^{4}\right)+z^{-2-4 L} b\left(1 / z^{4}\right)+z^{-3-4 L} a\left(1 / z^{4}\right) .
\end{aligned}
$$


We then need to find the polynomials $a(z)$ and $b(z)$. Substituting in the PR equations $(3,5,6)$, we have

$$
\begin{aligned}
8 a\left(z^{4}\right) a\left(1 / z^{4}\right)+8 b\left(z^{4}\right) b\left(1 / z^{4}\right) & + \\
\sum_{\forall n \in\{0,3,4,7\}} H_{n}(z) H_{n}(1 / z) & =4 \\
(4-\jmath 4)\left(a\left(z^{4}\right) a\left(1 / z^{4}\right)-b\left(z^{4}\right) b\left(1 / z^{4}\right)\right) & + \\
\sum_{\forall n \in\{0,3,4,7\}} H_{n}(\jmath z) H_{n}(1 / z) & =0, \\
(4+\jmath 4)\left(a\left(z^{4}\right) a\left(1 / z^{4}\right)-b\left(z^{4}\right) b\left(1 / z^{4}\right)\right) & + \\
\sum_{\forall n \in\{0,3,4,7\}} H_{n}(-\jmath z) H_{n}(1 / z) & =0 .
\end{aligned}
$$

Notice that substituting in equation (4) results in the left hand side identically zero $\forall z$. Then, collecting terms of equation (29), and adding equations (30) and (31), we obtain

$$
\begin{aligned}
8 a\left(z^{4}\right) a\left(1 / z^{4}\right)+8 b\left(z^{4}\right) b\left(1 / z^{4}\right) & =4-\sum_{\forall n \in\{0,3,4,7\}} H_{n}(z) H_{n}(1 / z) \\
8 a\left(z^{4}\right) a\left(1 / z^{4}\right)-8 b\left(z^{4}\right) b\left(1 / z^{4}\right) & =-\sum_{\forall n \in\{0,3,4,7\}} H_{n}(1 / z)\left(H_{n}(\jmath z)+H_{n}(-\jmath z)\right) .
\end{aligned}
$$

Written differently, we have

$$
\begin{array}{r}
16 a\left(z^{4}\right) a\left(1 / z^{4}\right)= \\
4-\sum_{\forall n \in\{0,3,4,7\}}\left(H_{n}(z)+H_{n}(\jmath z)+H_{n}(-\jmath z)\right) H_{n}(1 / z), \\
16 b\left(z^{4}\right) b\left(1 / z^{4}\right)= \\
4-\sum_{\forall n \in\{0,3,4,7\}}\left(H_{n}(z)-H_{n}(\jmath z)-H_{n}(-\jmath z)\right) H_{n}(1 / z) .
\end{array}
$$


Substituting equations $(21,24)$ in equations $(32,33)$ and simplifying, we obtain

$$
\begin{aligned}
4 a\left(z^{4}\right) a\left(1 / z^{4}\right) & =1-4 H_{00}\left(z^{4}\right) H_{00}\left(1 / z^{4}\right), \\
4 b\left(z^{4}\right) b\left(1 / z^{4}\right) & =1-4 H_{01}\left(z^{4}\right) H_{01}\left(1 / z^{4}\right) .
\end{aligned}
$$

The polynomials $a(z)$ and $b(z)$ are obtained via spectral factorization. The filters $\left\{h_{1}, h_{2}, h_{5}, h_{6}\right\}$ are then obtained using $a(z)$ and $b(z)$ as polyphase components, following equations $(26,27,28)$. The filter structure imposed by polynomials $a(z)$ and $b(z)$ guarantees (anti-)symmetry.

\subsection{Finding Filters $\left\{h_{1}, h_{2}, h_{5}, h_{6}\right\}$ With Length $4 L+2$}

In the case of length $h_{0}=4 L+2$, symmetric filter $h_{2}$ is written in terms of $a(z)$ and $b(z)$ slightly differently from the case outlined in Subsection 3.3. Then $H_{2}(z)$ is now expanded in $a(z)$ and $b(z)$ as follows:

$$
H_{2}(z)=a\left(z^{4}\right)+z^{-1-4 L} a\left(1 / z^{4}\right)+z^{-2} b\left(z^{4}\right)+z^{-3-4 L} b\left(1 / z^{4}\right) .
$$

Proceeding as in Section 3.3, we express the polynomials $a(z)$ and $b(z)$ in terms of the $H_{0}(z)$ polyphase components $H_{00}(z)$ and $H_{02}(z)$ :

$$
\begin{aligned}
4 a\left(z^{4}\right) a\left(1 / z^{4}\right) & =1-4 H_{00}\left(z^{4}\right) H_{00}\left(1 / z^{4}\right), \\
4 b\left(z^{4}\right) b\left(1 / z^{4}\right) & =1-4 H_{02}\left(z^{4}\right) H_{02}\left(1 / z^{4}\right) .
\end{aligned}
$$

Bandpass filter $H_{2}(z)$ is known from equation (36). Similar to Section 3.3, the filters $\left\{h_{1}, h_{5}, h_{6}\right\}$ are then obtained using $a(z)$ and $b(z)$ as polyphase components as follows:

$$
\begin{aligned}
& H_{1}(z)=-a\left(z^{4}\right)+z^{-1} a\left(z^{4}\right)-z^{-2-4 L} b\left(1 / z^{4}\right)+z^{-3-4 L} b\left(1 / z^{4}\right) \\
& H_{5}(z)=-a\left(z^{4}\right)+z^{-1} a\left(z^{4}\right)+z^{-2-4 L} b\left(1 / z^{4}\right)-z^{-3-4 L} b\left(1 / z^{4}\right) \\
& H_{6}(z)=-a\left(z^{4}\right)-z^{-1} a\left(z^{4}\right)+z^{-2-4 L} b\left(1 / z^{4}\right)+z^{-3-4 L} b\left(1 / z^{4}\right) .
\end{aligned}
$$




\section{Examples}

In this section, we consider examples of tight frame $M=4$ (anti-)symmetric filterbanks satisfying PR conditions. The filterbank is obtained starting with the lowpass filter $h_{0}$. The filter $h_{0}$ is obtained using the Taylor polynomial method described in Section 3.1. Given $h_{0}$, three more filters $\left\{h_{3}, h_{4}, h_{7}\right\}$ are directly obtained by modulating the lowpass filter $h_{0}$. In order to obtain the remaining four filters, we first compute polynomials $a(z)$ and $b(z)$ from spectral factorization equations $(34,35)$ or $(37,38)$, depending on the length of $h_{0}$. Then the filters $\left\{h_{1}, h_{2}, h_{5}, h_{6}\right\}$ are obtained as described in equations (25) or (36), and their modulations. In the following examples we will list only two of the eight filters, from which the remaining filters are obtained by modulation. All lowpass filters discussed in the examples possess dyadic coefficients.

\subsection{Example I: Case $K_{0}=4, K_{\min }=1$}

In this example, we seek a lowpass filter with approximation order $K_{0}=4$ and minimum number of vanishing moments $K_{\min }=1$ for each wavelet. The resulting lowpass filter is of length 14 , with Sobolev smoothness $\nu_{2}=4$. Since $K_{0}$ in this case is even, we use condition (19) to obtain the lowpass filter with $K=2$. In this particular case, since $K_{\min }=1$ the polynomial $A(x)$ is of zero degree, as is $Q_{0}(z)$, and $k=1$ in (14), resulting in the filter given in (42). We thus proceed to design the filterbank as outlined in equations $(37,38)$. Notice that the length of a lowpass symmetric orthogonal filter with $M=4$, and with $K_{0}=4$ is 24 , as compared with the lowpass filter in this example with length 14 only. The bandpass filter $h_{2}$ was found via spectral factorization of 
the polyphase components of $H_{0}(z)$. Filters $H_{0}(z)$ and $H_{2}(z)$ are given by:

$$
\begin{aligned}
H_{0}(z)= & \frac{1}{2^{8}}\left(1+z^{-1}+z^{-2}+z^{-3}\right)^{4}\left(1+z^{-1}\right) \\
H_{2}(z)= & \left(1-z^{-1}\right)^{2}\left(1+z^{-1}\right)\left(1+z^{-2}\right)^{2} Q_{2}(z) \\
Q_{2}(z)= & 0.0136\left(1+z^{-6}\right)+0.0079 z^{-1}\left(1+z^{-4}\right)+ \\
& 0.0188 z^{-2}\left(1+z^{-2}\right)-0.2375 z^{-3}
\end{aligned}
$$

The filters $\left\{h_{3}, h_{4}, h_{7}\right\}$ are obtained by modulating $h_{0}$, as shown in equations (22-24). The filters $\left\{h_{1}, h_{5}, h_{6}\right\}$ are obtained by modulating $h_{2}(26-28)$. The coefficients of filters $h_{0}$ and $h_{2}$ are given in Table 1 . Norm of $h_{0}$ is given by $\left\|h_{0}\right\|=0.6948$, while the norm of the bandpass filter $h_{2}$ is given by $\left\|h_{2}\right\|=0.7192$. In Fig. 1 we show the limit functions. The limit functions approximation order and vanishing moments for this filterbank are given by $\left\{K_{i}\right\}_{i=0}^{7}=\{4,1,2,5,4,3,2,5\}$. Filters $\left\{h_{0}, h_{2}, h_{4}, h_{6}\right\}$ are symmetric, while filters $\left\{h_{1}, h_{3}, h_{5}, h_{7}\right\}$ are antisymmetric. While we have $H_{7}(z)=H_{0}(-z)$, the additional zero of $H_{0}(z)$ at $z=-1$ leads to $K_{7}>K_{0}$. Filters' magnitudes are shown in Fig. 2.

Compare with the case of $M=4$ symmetric orthogonal filters [22], where the lowpass filter with 12 coefficients provides only $K_{0}=2$, and its scaling function lacks the smoothness (with $\nu_{2} \approx 1.1224$ ) associated with the scaling function generated by this example's filters. Fig. 3 shows the limit functions' frequency response at the second stage. The top figure shows the frequency response of symmetric orthogonal $M=4$ limit functions $\left(K_{0}=2\right)$ with underlying filters of length 12 [22]. The bottom figure shows tight frame symmetric limit functions second stage frequency response due to the tight frame filters discussed in this example. The tight frame limit functions enjoy 


\begin{tabular}{rrr}
\hline$n$ & $h_{0}(n)$ & $h_{2}(n)$ \\
\hline 0 & $1 / 2^{8}$ & -0.013559146419 \\
1 & $5 / 2^{8}$ & 0.005626751342 \\
2 & $14 / 2^{8}$ & 0.024465770518 \\
3 & $30 / 2^{8}$ & -0.261945210417 \\
4 & $51 / 2^{8}$ & -0.253665936755 \\
5 & $71 / 2^{8}$ & 0.261598331831 \\
6 & $84 / 2^{8}$ & 0.237479439900 \\
7 & $84 / 2^{8}$ & 0.237479439900 \\
8 & $71 / 2^{8}$ & 0.261598331831 \\
9 & $51 / 2^{8}$ & -0.253665936755 \\
10 & $30 / 2^{8}$ & -0.261945210417 \\
11 & $14 / 2^{8}$ & 0.024465770518 \\
12 & $5 / 2^{8}$ & 0.005626751342 \\
13 & $1 / 2^{8}$ & -0.013559146419 \\
\hline
\end{tabular}

Table 1: Example I, filters $h_{0}$ and $h_{2}$ for case $K_{0}=4$ and $K_{\min }=1$, and length $h_{0}=14$. 

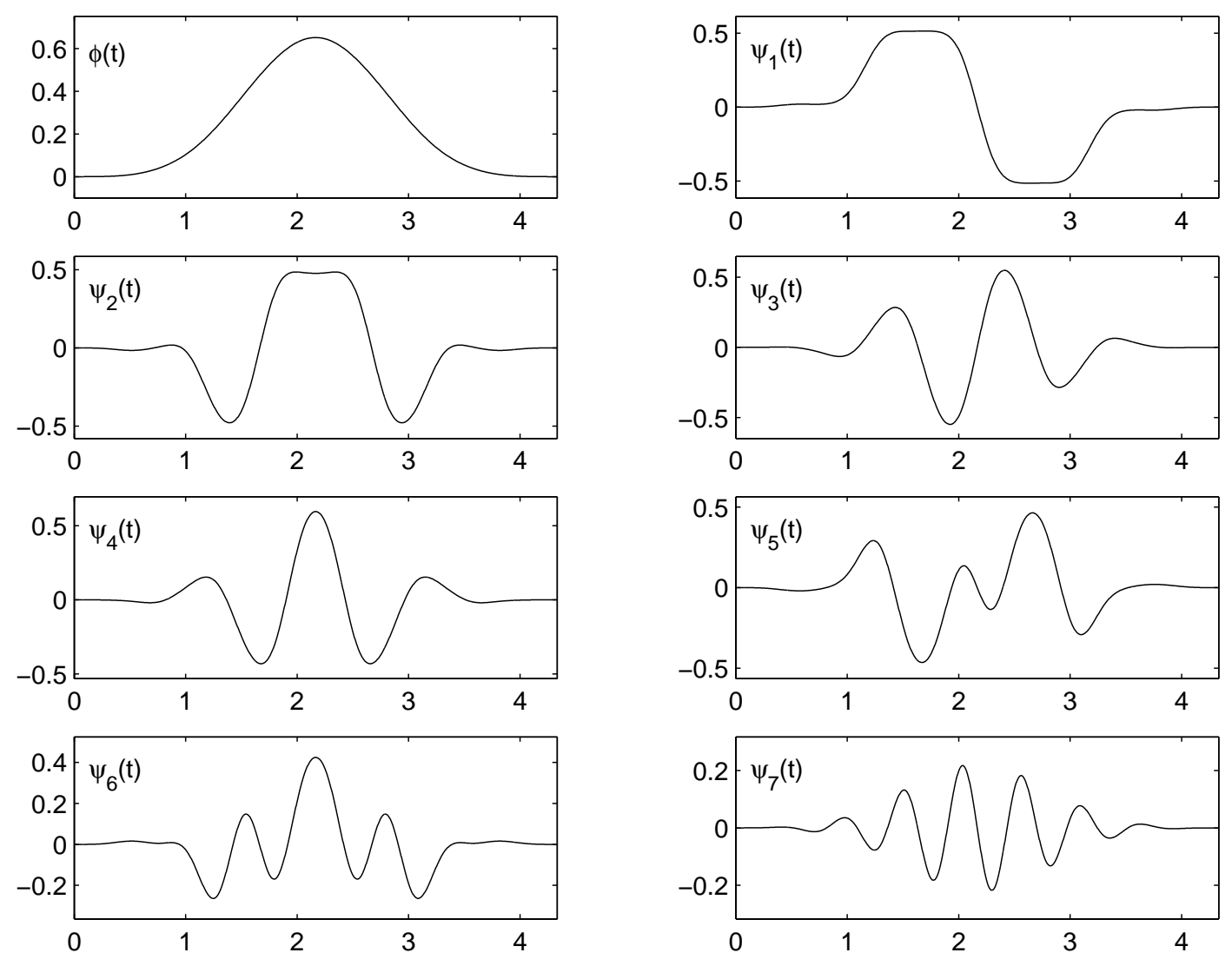

Figure 1: Example I, limit functions with $K_{0}=4, K_{\min }=1$ and $\nu_{2}=4$. 


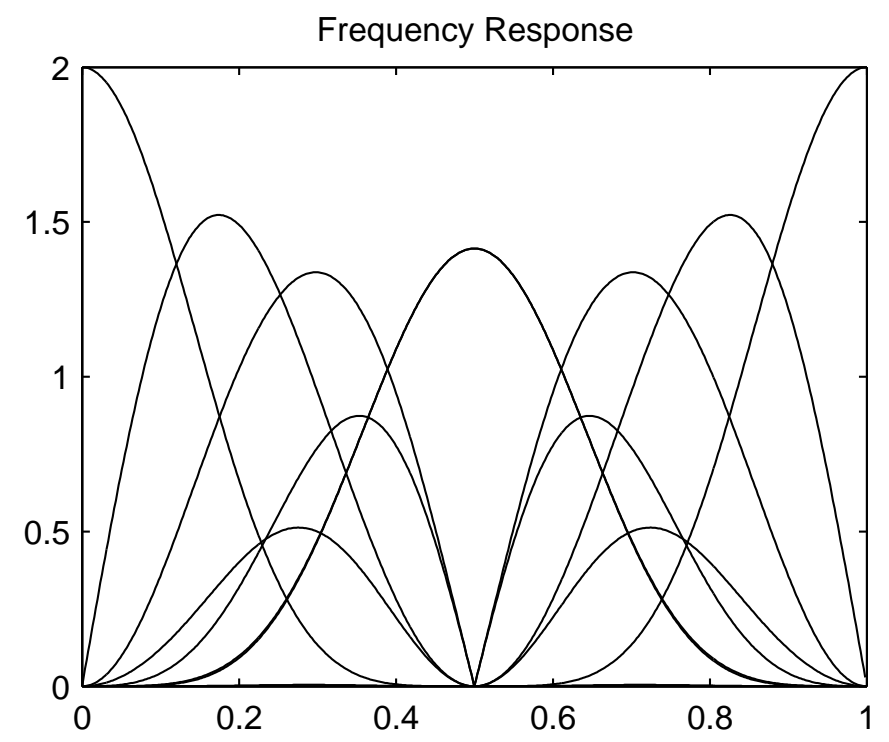

Figure 2: Example I, filterbank corresponding to limit functions of Fig. 1

an enhanced frequency selectivity while maintaining smoothness.

\subsection{Example II: Case $K_{0}=7, K_{\min }=2$}

In this example we consider the case of approximation order $K_{0}=7$ with a minimum number of vanishing moments $K_{\min }=2$. The filters are of minimum length, with 24 coefficients. Since $K_{0}$ in this case is odd, the lowpass filter $h_{0}$ is obtained starting with a Taylor polynomial with $K=3$ (recall $K_{0}=2 K+1$ ), as shown in (20). The polynomial $A(x)$ in this case is of degree one given by

$$
A(x)=8+140 x
$$

with $Q_{0}(z)$ obtained from

$$
\begin{aligned}
Q_{0}(z) & =A\left(\frac{-z^{-1}+2-z}{4}\right) \\
& =-35 z^{-1}+78-35 z .
\end{aligned}
$$



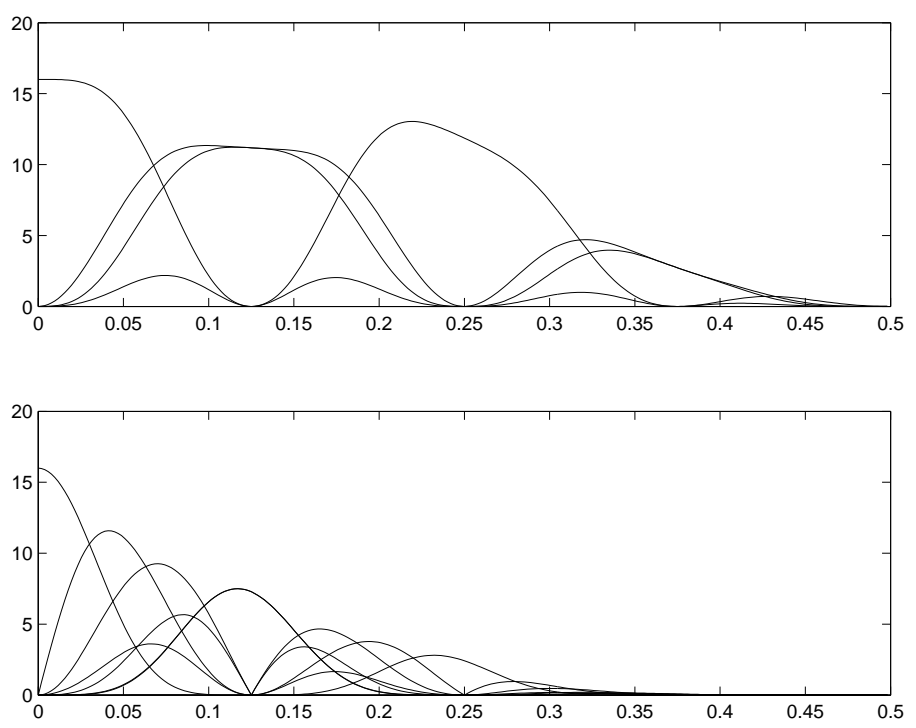

Figure 3: Example I: second stage frequency responses of orthogonal $M=4$ and $K_{0}=2$ symmetric limit functions [22] (top), and $M=4$ and $K_{0}=4$ tight frame limit functions (bottom). Notice how the latter enjoy an improved frequency selectivity.

This leads to filters $\left\{h_{3}, h_{4}, h_{7}\right\}$, as described in equations (22-24). To find the filters $\left\{h_{1}, h_{2}, h_{5}, h_{6}\right\}$ we first find $h_{1}$ from the $H_{0}(z)$ polyphase components $H_{00}(z)$ and $H_{01}(z)$ using spectral factorization equations $(34,35)$. The resulting scaling function has $\nu_{2} \approx 4.7352$. Filters $\left\{h_{0}, h_{1}, h_{4}, h_{5}\right\}$ are symmetric, while filters $\left\{h_{2}, h_{3}, h_{6}, h_{7}\right\}$ are antisymmetric. Filters $H_{0}(z)$ and $H_{1}(z)$ are listed in equations $(44,45)$.

$$
\begin{aligned}
H_{0}(z)= & \frac{1}{2^{16}}\left(1+z^{-1}+z^{-2}+z^{-3}\right)^{7}\left(35 z^{-2}-78 z^{-1}+35\right) \\
H_{1}(z)= & \frac{1}{4^{2}}\left(1-z^{-1}\right)^{2}\left(1+z^{-1}\right)^{2}\left(1+z^{-2}\right)^{3} Q_{1}(z), \\
Q_{1}(z)= & 0.0085\left(1+z^{-13}\right)+0.0184 z^{-1}\left(1+z^{-11}\right)-0.2626 z^{-2}\left(1+z^{-9}\right)- \\
& 0.2649 z^{-3}\left(1+z^{-7}\right)+0.7149 z^{-4}\left(1+z^{5}\right)+0.6778 z^{-5}\left(1+z^{-3}\right)+ \\
& 1.4053 z^{-6}\left(1+z^{-1}\right) .
\end{aligned}
$$


Notice that for the same length as the filters in this example, orthogonality (with $M=4$ ) leads to $K_{0}$ reducing from 7 to 4 .
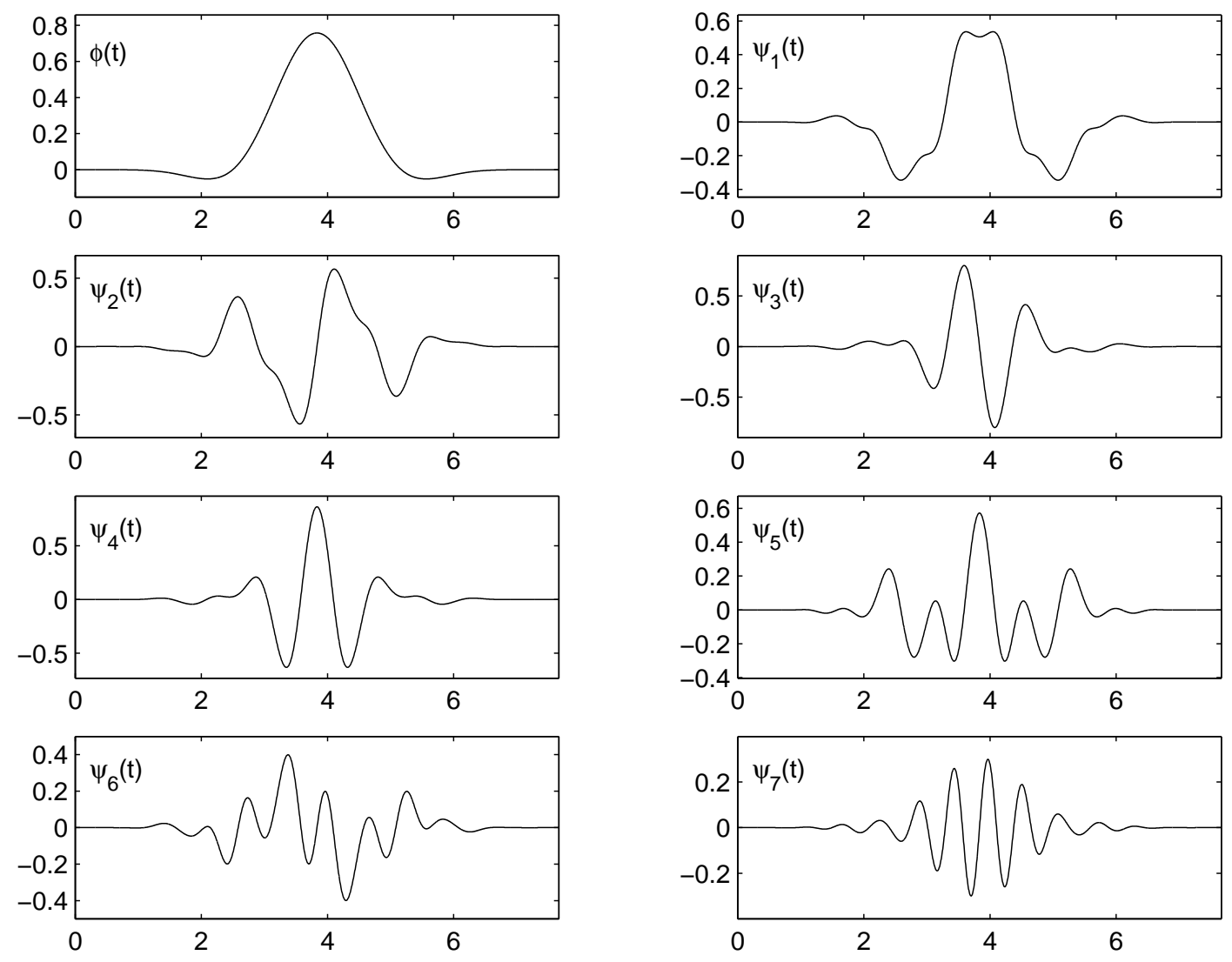

Figure 4: Example II, limit functions with $\left\{K_{i}\right\}_{i=0}^{7}=\{7,2,3,7,8,4,3,7\}$, and $\nu_{2} \approx 4.7352$

The resulting limit functions have the following set of approximation order and vanishing moments: $\left\{K_{i}\right\}_{i=0}^{7}=\{7,2,3,7,8,4,3,7\}$. Table 2 lists the coefficients of filters $h_{0}$ and $h_{1}$. The resulting limit functions are shown in Fig. 4. The norm of $h_{0}$ is given by $\left\|h_{0}\right\|=0.7832$, while that of $h_{1}$ is given by $\left\|h_{1}\right\|=0.6217$. Filterbank's frequency response is depicted in Fig. 5 . 


\begin{tabular}{rrr}
\hline$n$ & $h_{0}(n)$ & $h_{1}(n)$ \\
\hline 0 & $-35 / 2^{16}$ & 0.000529322 \\
1 & $-167 / 2^{16}$ & 0.001147920 \\
2 & $-469 / 2^{16}$ & -0.015886124 \\
3 & $-1001 / 2^{16}$ & -0.015410684 \\
4 & $-1533 / 2^{16}$ & 0.027208254 \\
5 & $-1561 / 2^{16}$ & 0.023509020 \\
6 & $-371 / 2^{16}$ & 0.164283530 \\
7 & $2849 / 2^{16}$ & 0.161013769 \\
8 & $8114 / 2^{16}$ & 0.074187926 \\
9 & $14602 / 2^{16}$ & 0.082049484 \\
10 & $20734 / 2^{16}$ & -0.255103829 \\
11 & $24374 / 2^{16}$ & -0.247528586 \\
12 & $24374 / 2^{16}$ & -0.247528586 \\
13 & $20734 / 2^{16}$ & -0.255103829 \\
14 & $14602 / 2^{16}$ & 0.082049484 \\
15 & $8114 / 2^{16}$ & 0.074187926 \\
16 & $2849 / 2^{16}$ & 0.161013769 \\
17 & $-371 / 2^{16}$ & 0.164283530 \\
18 & $-1561 / 2^{16}$ & 0.023509020 \\
19 & $-1533 / 2^{16}$ & 0.027208254 \\
20 & $-1001 / 2^{16}$ & -0.015410684 \\
21 & $-469 / 2^{16}$ & -0.015886124 \\
22 & $-167 / 2^{16}$ & 0.001147920 \\
23 & $-35 / 2^{16}$ & 0.000529322 \\
\hline & &
\end{tabular}

Table 2: Example II, coefficients of filters $h_{0}$ and $h_{1}$ for case $K_{0}=7$ and $K_{\min }=2$. 


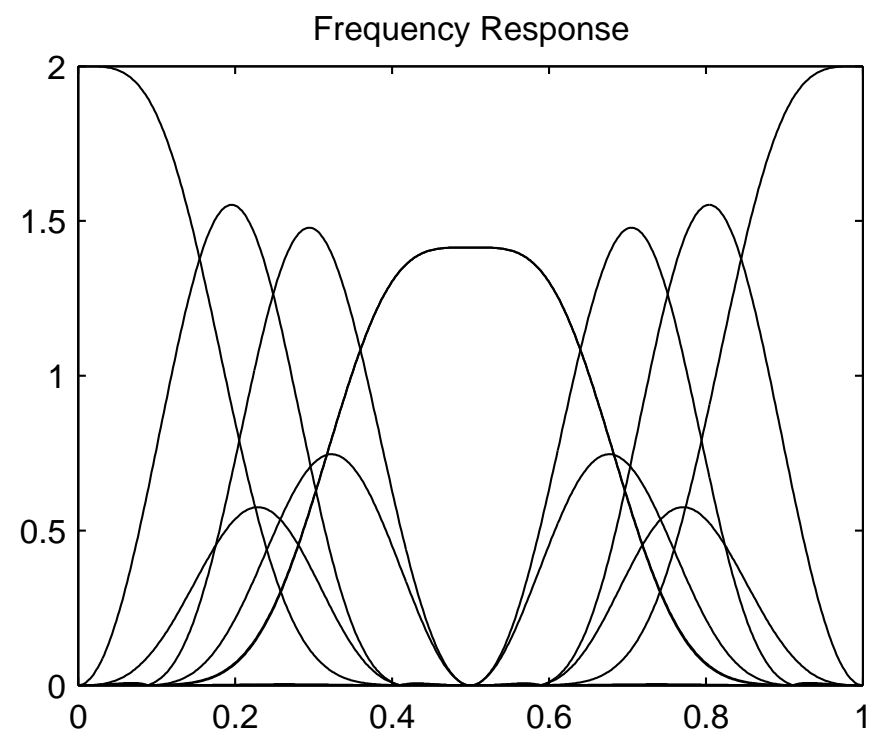

Figure 5: Example II, filters associated with limit functions of Fig. 4

\subsection{Example III: Case $K_{0}=5, K_{\min }=2$}

We seek a lowpass filter with $K_{0}=5$ and $K_{\min }=2$. With $K_{0}$ odd we seek to generate a Taylor polynomial using expression (20). The additional requirement $K_{\min }=2$ leads to the polynomial $A(x)$ of length 2, as follows:

$$
A(x)=8+100 x,
$$

and we have

$$
\begin{aligned}
Q_{0}(z) & =A\left(\frac{-z^{-1}+2-z}{4}\right) \\
& =-25 z^{-1}+58-25 z .
\end{aligned}
$$


The bandpass filter $h_{1}$ is found from spectral factorization equations $(34,35)$. The resulting filters $h_{0}$ and $h_{1}$ are given as follows:

$$
\begin{aligned}
H_{0}(z)= & \frac{1}{2^{12}} z^{-1}\left(1+z^{-1}+z^{-2}+z^{-3}\right)^{5}\left(25-58 z^{-1}+25 z^{-2}\right) \\
H_{1}(z)= & \frac{1}{8}\left(1-z^{-1}\right)^{2}\left(1+z^{-1}\right)^{3}\left(1+z^{-2}\right)^{3} Q_{1}(z) \\
Q_{1}(z)= & 0.0609\left(1+z^{-8}\right)+0.0091 z^{-1}\left(1+z^{-6}\right)-0.1613 z^{-2}\left(1+z^{-4}\right)+ \\
& 0.0913 z^{-3}\left(1+z^{-2}\right)-0.8356 z^{-4} .
\end{aligned}
$$

The smoothness coefficient associated with the lowpass filter $h_{0}$ is $\nu_{2} \approx$ 3.1245. The filters obtained in this example are minimum length, with length $h_{0}=18$, in addition to a one sample shift, and agreeing with inequality (11). For the bandpass filter we have length $h_{1}=20$. Filters $\left\{h_{0}, h_{1}, h_{4}, h_{5}\right\}$ are symmetric, while filters $\left\{h_{2}, h_{3}, h_{6}, h_{7}\right\}$ are antisymmetric.

We obtain the following limit functions approximation order and vanishing moments for this case: $\left\{K_{i}\right\}_{i=0}^{7}=\{5,2,3,5,6,4,3,5\}$. Table 3 lists the resulting coefficients for filters $h_{0}$ and $h_{1}$. The norm of the lowpass filter $h_{0}$ is found to be $\left\|h_{0}\right\|=0.8444$, while that of filter $h_{1}$ is found to be $\left\|h_{1}\right\|=0.5358$. The resulting limit functions are shown in Fig. 6, and the corresponding filterbank's frequency response is given in Fig. 7.

\section{Application Example}

In this section we present a simple denoising application example for the case of 1-D signals. We compare with other published wavelets, including orthogonal and symmetric tight frame wavelets with $M=2$. Since we are comparing the performance of filters with different scaling factors and numbers of bands, our comparison criteria consist of having identical $K_{0}$ for 


\begin{tabular}{rrr}
\hline$n$ & $h_{0}(n)$ & $h_{1}(n)$ \\
\hline 0 & 0 & 0.007606335 \\
1 & $-25 / 2^{12}$ & 0.008745493 \\
2 & $-67 / 2^{12}$ & -0.011415913 \\
3 & $-110 / 2^{12}$ & 0 \\
4 & $-130 / 2^{12}$ & -0.127270113 \\
5 & $30 / 2^{12}$ & -0.119271674 \\
6 & $370 / 2^{12}$ & -0.078948864 \\
7 & $858 / 2^{12}$ & -0.112057444 \\
8 & $1430 / 2^{12}$ & 0.231721222 \\
9 & $1740 / 2^{12}$ & 0.200890958 \\
10 & $1740 / 2^{12}$ & 0.200890958 \\
11 & $1430 / 2^{12}$ & 0.231721222 \\
12 & $858 / 2^{12}$ & -0.112057444 \\
13 & $370 / 2^{12}$ & -0.078948864 \\
14 & $30 / 2^{12}$ & -0.119271674 \\
15 & $-130 / 2^{12}$ & -0.127270113 \\
16 & $-110 / 2^{12}$ & 0 \\
17 & $-67 / 2^{12}$ & -0.011415913 \\
18 & $-25 / 2^{12}$ & 0.008745493 \\
19 & 0 & 0.007606335 \\
\hline & &
\end{tabular}

Table 3: Example III, filters $h_{0}$ and $h_{1}$ coefficients for the case $K_{0}=5$, and $K_{\min }=2$. 

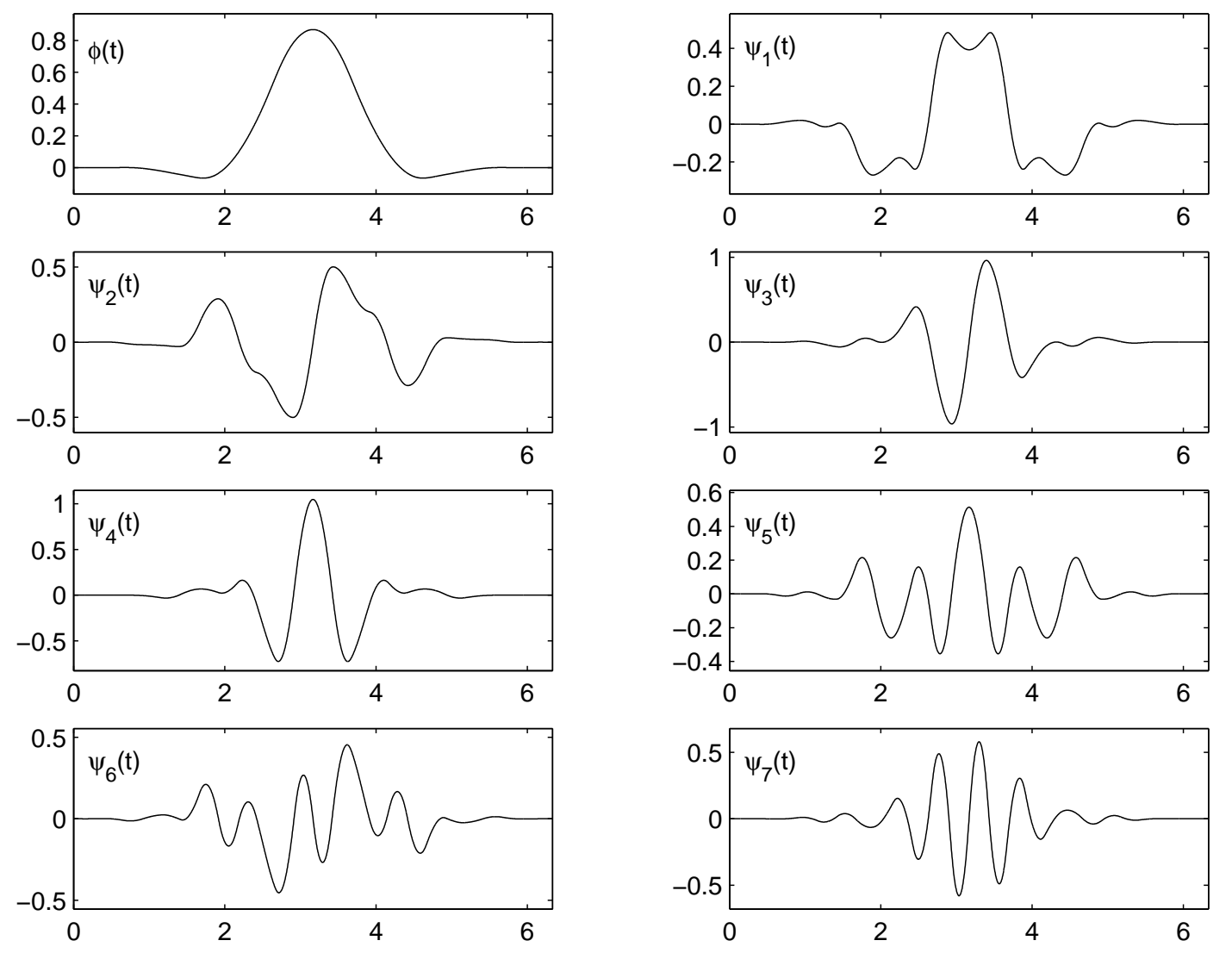

Figure 6: Example III, limit functions with $\left\{K_{i}\right\}_{i=0}^{7}=\{5,2,3,5,6,4,3,5\}$, and $\nu_{2} \approx$ 3.1245 . 


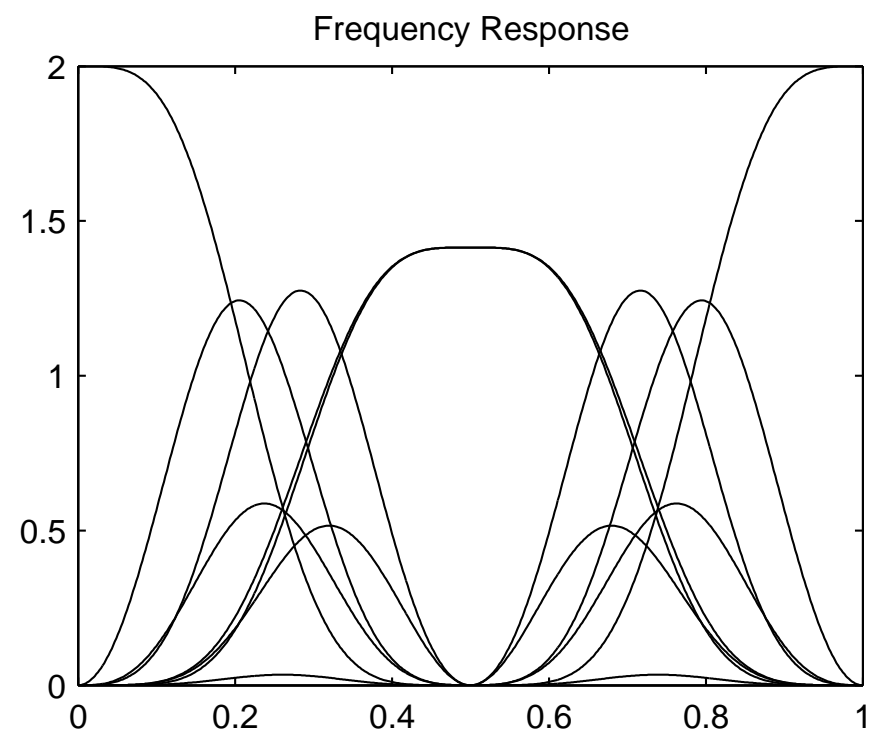

Figure 7: Example III, filterbank resulting in the limit functions illustrated in Fig. 6

filterbanks, and the length of the output of the last stage is 64 samples. For the case of $M=2$ and input signal $x(n)$ of length 4096 this translates into 6 stages, while for the case $M=4$ we use 3 stages. For the proposed wavelets, we use the filters with $K_{0}=5$. Following [50], we generate a signal from WaveLab software package [51] ('Doppler') of length 4096, normalized to one, and with additive zero mean Gaussian noise with standard deviation 0.125. We use hard threshold for all wavelets with a global threshold $T$. For the tight frame wavelets we additionally take into account the non-unity norms of the filters, multiplying $T$ by the corresponding subband norm prior to denoising. The threshold was applied to every subband except the lowpass subband. We compare our wavelets with Daubechies orthogonal wavelet [1] and 4-band symmetric wavelets with $M=2[16,18]$, all with $K_{0}=5$. For the frames we consider the cases with $K_{\min }=1$ and $K_{\min }=2$ for $M=2$ 
and $M=4$. Fig. 8 depicts the denoising error from the five wavelets. It is

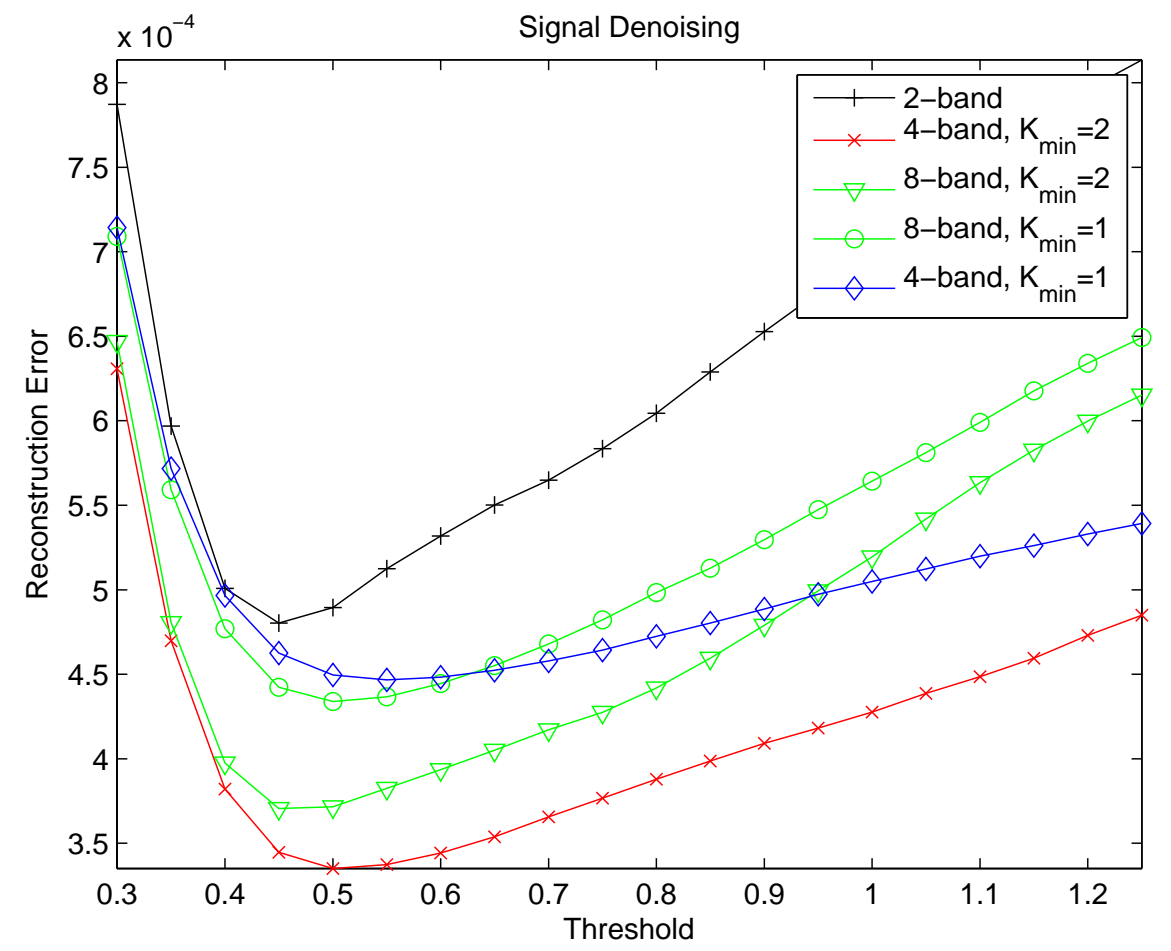

Figure 8: Denoising performance of proposed wavelets (' $\nabla$ ' for $K_{\min }=2$, and ' $\circ$ ' for $\left.K_{\min }=1\right)$ compared with published filters. The proposed wavelets performance is comparable with the more redundant 4 -band wavelets in $[16,18]\left(K_{\min }=2\left({ }^{\prime} \times\right)\right.$ and $K_{\min }=1$ $\left.\left(\diamond^{\prime}\right)\right)$.

known [3] that generally the more redundant wavelets lead to an improved reconstruction error. Referring to Fig. 8, the proposed wavelets performance is comparable with the $M=2$ counterparts for the case $K_{\min }=1$, and slightly inferior in the case of $K_{\min }=2$. For a signal of length 4096 the $M=2$ wavelets implemented with six stages yield 12160 coefficients, while the less redundant proposed wavelets yield 9472 wavelet coefficients and three stages, 
about $22 \%$ less redundant and thus computationally less demanding.

\section{Discussion}

The family of filterbanks presented in this article is a particular case of 1D filterbanks discussed in [38]. The resulting filterbanks are more frequency selective than their $M=2$ counterparts, and their tight frame property allows for smoother scaling functions and wavelets as compared with their $M=4$ orthogonal counterparts $[22,52]$. We list in table 4 the smoothness $\nu_{2}$ for various values of $K_{0}=2, \ldots, 5$. For the tight frame case, we consider the smoothness of the lowpass filters with the corresponding $K_{\min }=1$ for the bandpass/highpass filters. We note that for the case $K_{0}=3$ a second

\begin{tabular}{rrr}
\hline$K_{0}$ & $\nu_{2}$, Tight frame & $\nu_{2}$, Orthogonal \\
\hline 2 & 2.00 & 1.1224 \\
3 & 2.75 & 1.1965 \\
4 & 4.00 & 1.1182 \\
5 & 4.50 & 1.0164 \\
\hline
\end{tabular}

Table 4: Smoothness of scaling functions for various values of $K_{0}$ corresponding to $M=4$ for the cases of tight frame symmetric filterbanks and the symmetric orthogonal ones.

lowpass even length filter satisfying condition (8) exists with $K_{\min }=1$ and $\nu_{2} \approx 1.9575$. The lowpass filter design method discussed in Section 3.1 can easily be extended to the odd-length filters with minor modifications. The limited smoothness shown by the orthogonal filters in Table 4 may be attributed to the fact that the orthogonality requirement is such that the factor $Q_{0}(z)$ is of higher degree than the frame counterparts, limiting the effect of the factor $\left(1+z^{-1}+z^{-2}+z^{-3}\right)^{K_{0}}$. 


\section{Conclusion}

In this paper we examined the construction of a tight frame (anti-)symmetric filterbank family for the case of scaling factor $M=4$. The filters are simple to design, and the accompanying limit functions are highly smooth. The lowpass filter $h_{0}$ is obtained via simple truncation of Taylor series governed by the parameters $K_{0}$ and $K_{m i n}$, then three of the wavelet filters are obtained by modulating $h_{0}$. The remaining filters are found by obtaining two polynomials $a(z)$ and $b(z)$ from spectral factorization. For future work we plan to explore alternative symmetric dual and sibling frame filterbanks with $M=4$.

\section{Acknowledgement}

The author would like to thank Ivan Selesnick for constructive conversations and the reviewers for suggestions that have significantly improved the quality and readability of the paper and for pointing at related references.

\section{References}

[1] I. Daubechies. Ten Lectures On Wavelets. SIAM, 1992.

[2] C. Blatter. Wavelets, A Primer. A. K. Peters, 1998.

[3] H. Bölcskei, F. Hlawatsch, and H. G. Feichtinger. Frame-theoretical analysis of oversampled filter banks. IEEE Trans. Signal Process., 46(12):3256-3268, December 1998.

[4] Z. Cvetković and M. Vetterli. Oversampled filter banks. IEEE Trans. Signal Process., 46(5):1245-1255, May 1998. 
[5] A. Ron and Z. Shen. Construction of compactly supported affine frames in $L_{2}\left(R^{d}\right)$. In K.S. Lau, editor, Advances in Wavelets. Springer Verlag, 1998.

[6] S. Pei and M. Yeh. An introduction to discrete finite frames. IEEE Signal Process. Mag., pages 84-96, November 1997.

[7] B. Han. On dual wavelet tight frames. Appl. Comput. Harmon. Anal., (4):380-413, 1997.

[8] I. Daubechies and B. Han. Pairs of dual wavelet frames from any two refinable functions. Constr. Approx., 20(3):325-352, 2004.

[9] J. Kovačević and A. Chebira. Life beyond bases: The advent of frames (part I). IEEE Signal Process. Mag., 24(4):86-104, July 2007.

[10] J. Kovačević and A. Chebira. Life beyond bases: The advent of frames (part II). IEEE Signal Process. Mag., 24(5):115-125, Sept. 2007.

[11] I. W. Selesnick. Balanced multiwavelet bases based on symmetric FIR filters. IEEE Trans. Signal Process., 48(1):184-191, June 2000.

[12] I. W. Selesnick. Smooth wavelets tight frames with zero moments. Appl. Comput. Harmon. Anal., 10(2):163-181, March 2001.

[13] A. Petukhov. Symmetric framelets. Constr. Approx., 19(2):309-328, January 2003.

[14] Q. Jiang. Parameterizations of masks for tight affine frames with two symmetric/antisymmetric generators. Adv. Comput. Math., 18:247-268, February 2003. 
[15] I. W. Selesnick and F. Abdelnour. Symmetric wavelet tight frames with two generators. Appl. Comput. Harmon. Anal., 17(2):211-225, September 2004 .

[16] C. K. Chui and W. He. Compactly supported tight frames associated with refinable functions. Appl. Comput. Harmon. Anal., 8(3):293-319, May 2000.

[17] C. K. Chui, W. He, and J. Stöckler. Compactly supported tight and sibling frames with maximum vanishing moments. Appl. Comput. Harmon. Anal., 13:224-262, November 2002.

[18] F. Abdelnour and I. W. Selesnick. Symmetric nearly shift invariant tight frame wavelets. IEEE Trans. Signal Process., 53(1), January 2005.

[19] B. Han and Q. Mo. Symmetric MRA tight wavelet frames with three generators and high vanishing moments. Appl. Comput. Harmon. Anal., 18(1):67-93, January 2005.

[20] F. Abdelnour. Symmetric tight frames with shifted wavelets. In Wavelets XI Proc. of SPIE, volume 5914, pages 133-140, San Diego, 31 July - 4 August 2005.

[21] F. Abdelnour. Tight frame various lengths filters. In World Multi-Conf. on Systemics, Cybernetics and Informatics, Orlando, 27-30 July 2003.

[22] B. Han. Symmetric orthonormal scaling functions and wavelets with dilation factor 4. Adv. in Comput. Math., (8):221-247, 1998. 
[23] S. Oraintara, T. D. Tran, P. N. Heller, and T. Q. Nguyen. Lattice structure for regular paraunitary linear-phase filterbanks and $M$-band orthogonal symmetric wavelets. IEEE Trans. Signal Process., 49(11):26592672, November 2001.

[24] A. K. Soman, P. P. Vaidyanathan, and T. Q. Nguyen. Linear phase paraunitary filter banks: theory, factorizations and designs. IEEE Trans. Signal Process., 41(12):3480-3496, December 1993.

[25] P. Steffen, P. Heller, R. A. Gopinath, and C. S. Burrus. Theory of regular $M$-band wavelet bases. IEEE Trans. Signal Process., 41(12):3497-3511, December 1993.

[26] S. Oraintara, T. D. Tran, and T. Q. Nguyen. A class of regular biorthogonal linear-phase filterbanks: theory, structure, and application in image coding. IEEE Trans. Signal Process., 51(12):3220-3235, December 2003.

[27] J.-C. Han and Z.-X. Cheng. The construction of $M$-band tight wavelet frames. In Proc. of Int. Conf. on Machine Learning and Cybernetics., volume 6, pages 3924-3927, 26-29 August 2004.

[28] T. Tanaka. A direct design of oversampled perfect reconstruction FIR filter banks. IEEE Trans. Signal Process., 54(8):3011-3022, August 2006.

[29] M. Ehler. Compactly supported multivariate pairs of dual wavelet frames obtained by convolution. Int. Jnl. Wavelets Multires. inform. Process., 6(2):1-26, 2008.

[30] I. Daubechies, B. Han, A. Ron, and Z. Shen. Framelets: MRA-based 
constructions of wavelet frames. Appl. Comput. Harmon. Anal., 14(1):146, 2003.

[31] F. Abdelnour. Design of 6-band tight frame wavelets with limited redundancy. In IEEE Int. Symp. Signal Process. Information Technology (ISSPIT), pages 133-137, Vancouver, August 27-30 2006.

[32] H. Park. Symbolic computation and signal processing. Jnl. Symb. Comput., 37:209-226, 2004.

[33] J. Lebrun and I. Selesnick. Gröbner bases and wavelet design. Jnl. Symb. Comput., (2):227-259, February 2004.

[34] B. Yang and Z. Jing. A simple method to build oversampled filter banks and tight frames. IEEE Trans. Image Process., 16(11):2682-2687, Nov. 2007.

[35] F. Abdelnour. Dual frame symmetric wavelets with limited redundancy. In Vivek K. Goyal, Manos Papadakis, and Dimitri Van De Ville, editors, Wavelets XIII, vol. 7446, page 744610, San Diego, 2009. SPIE.

[36] M. Ehler. On multivariate compactly supported bi-frames. Jnl. Fourier Anal. Appl., 13:511-532, 2007. 10.1007/s00041-006-6021-1.

[37] B. Han and H. Ji. Compactly supported orthonormal complex wavelets with dilation 4 and symmetry. Appl. Comput. Harmon. Anal., 26(3):422-431, 2009.

[38] A. Petukhov. Construction of symmetric orthogonal bases of wavelets 
and tight wavelet frames with integer dilation factor. Appl. Comput. Harmon. Anal., 17:198-210, 2004.

[39] O. Herrmann. On the approximation problem in nonrecursive digital filter design. IEEE Trans. Circuits and Systems, 18(3):411- 413, May 1971.

[40] B. Han. Symmetric orthonormal complex wavelets with masks of arbitrarily high linear-phase moments and sum rules. Adv. Comput. Math., 32:209-237, 2010.

[41] H. O. Kim, R. Y. Kim, and J. K. Lim. Internal structure of the multiresolution analyses defined by the unitary extension principle. Jnl. Approx. Theory, 154(2):140-160, October 2008.

[42] M. Ehler. The multiresolution structure of pairs of dual wavelet frames for a pair of Sobolev spaces. Jaen J. Approx., 2(2), 2010.

[43] M. Vetterli and J. Kovačević. Wavelets and Subband Coding. Prentice Hall, 1995.

[44] G. Strang and T. Nguyen. Wavelets and Filter Banks. WellesleyCambridge Press, 1996.

[45] O. Christensen. An Introduction to Frames and Riesz Bases. Birkhauser, Boston, 2003.

[46] C. S. Burrus, R. A. Gopinath, and H. Guo. Introduction to Wavelets and Wavelet Transforms. Prentice Hall, 1997. 
[47] C. Chui and Q. Sun. Affine frame decompositions and shift-invariant spaces. Appl. Comput. Harmon. Anal., 20:74-107, 2006.

[48] T. Eirola. Sobolev characterization of solutions of dilation equations. SIAM J. Math. Anal., 23:1015-1031, July 1992.

[49] H. Volkmer. On the regularity of wavelets. IEEE Trans. Info. Theory, 38:872-876, March 1992.

[50] I. W. Selesnick. A higher density discrete wavelet transform. IEEE Trans. Signal Process., 54(8):3039-3048, August 2006.

[51] D. Donoho, M. R. Duncan, X. Huo, and O. Levi. Wavelab 850, December 2005.

[52] F. Abdelnour. Wavelet Design Using Gröbner Basis Methods. PhD thesis, Polytechnic University, 2002. http://taco.poly.edu/farras. 